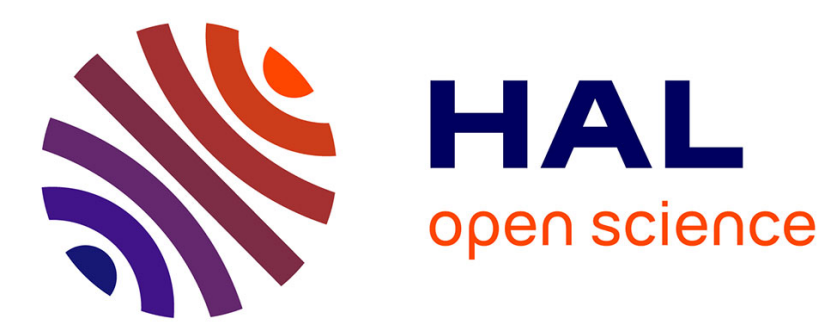

\title{
Concentric time: enabling context + focus visual analysis of architectural changes
}

Jean-Yves Blaise, Iwona Dudek

\section{To cite this version:}

Jean-Yves Blaise, Iwona Dudek. Concentric time: enabling context + focus visual analysis of architectural changes. 19th International Symposium on Methodologies for Intelligent Systems, Jun 2011, Warszawa, Poland. pp.632-641. halshs-00656010

\section{HAL Id: halshs-00656010 https://shs.hal.science/halshs-00656010}

Submitted on 3 Jan 2012

HAL is a multi-disciplinary open access archive for the deposit and dissemination of scientific research documents, whether they are published or not. The documents may come from teaching and research institutions in France or abroad, or from public or private research centers.
L'archive ouverte pluridisciplinaire HAL, est destinée au dépôt et à la diffusion de documents scientifiques de niveau recherche, publiés ou non, émanant des établissements d'enseignement et de recherche français ou étrangers, des laboratoires publics ou privés. 


\title{
Concentric time: enabling context + focus visual analysis of architectural changes
}

\author{
Jean-Yves Blaise ${ }^{1}$, Iwona Dudek ${ }^{1}$ \\ ${ }^{1}$ FRE 3315 CNRS/MCC, \\ 13288 Marseille Cedex 09, France \\ \{jyb, idu, @map.archi.fr\}
}

\begin{abstract}
In order to acquire and share a better understanding architectural changes, researchers face the challenge of modelling and representing events (cause and consequences) occurring in space and time, and for which assessments of doubts are vital. This contribution introduces a visualisation designed to facilitate reasoning tasks, in which a focus view on evidence about what happens to artefact $\lambda$ at time $\boldsymbol{t}$ is a complemented with a context view where successive spatial configurations of neighbouring artefacts, durations of changes, and punctual events are correlated and tagged with uncertainty markers.
\end{abstract}

\section{Introduction}

Analysing, representing, cross-examining what is really known about transformations occurring during the lifetime of historic artefacts is a research topic where issues specific to historic sciences often meet models and formalisms developed in computer science. Although largely outnumbered by self-evident applications of computer graphics, fruitful knowledge representation-oriented research efforts do exist on the modelling of such spatial dynamics.

But gaining insight (in the sense of [1]) into architectural changes is not only about circumscribing the relevant pieces of knowledge - i.e. abstracting relevant properties of the reality observed, and foreseeing possible processing. In historic sciences particularly, it is also basically about giving users means to shed a new light on their knowledge, to draw individual, self-achieved, maybe temporary conclusions - i.e. graphics as a discovery tool [2]. And indeed an effective way to support reasoning is to capitalize on the user's capacity to use vision to think [3].

Now what do we here need to reason about? Architectural changes occur in space, and in time - analysing changes will mean handling both dimensions. Changes may have implications on the shape of an artefact or not, anyway the evidence is liable to be distributed in space/time slots. Of course, visual solutions that help distributing and analysing information in space exist, either in 2D or 3D [4]. In parallel, other visual solutions may help us to distribute events in time, using the basic timeline paradigm, or more sophisticated concepts [5][6]. But architectural changes do not occur in space, on one hand, and in time, on another hand. If we want graphics to help us perform reasoning tasks, we need them to combine a spatial representation and a chronology 
representation". So-called "time sliders" (nested inside 2D or 3D interactive graphics) could be seen as an answer: they allow users to select a time slot and observe the corresponding spatial configuration. But what the user sees is one period at a time: in other words, a focus view. And beyond merely pointing out facts - artefact $\lambda$ changes at time $\boldsymbol{t}$-amplifying cognition [7] about architectural changes implies a teleological approach through which we connect facts about an artefact to a context - previous and following changes, things that happen in the neighbourhood, events that occur during that same period of time, etc. Besides a focus view, we need a context view where successive spatial configurations, durations of changes, punctual events, etc., are correlated. Finally, as usual in historic sciences, the evidence we base on is uncertain: efficient graphics should help visualising that uncertainty ${ }^{2}$.

In previous contributions, we focused on modelling issues, starting from two ends: the spatial bias (modelling the lifetime of artefacts as chains linking version to one another, with identification/classification issues) and the temporal bias (modelling key moments in the evolution of artefacts and processes of transformation in a cause + consequence approach). As a result, we developed solutions ranging from knowledge visualisation to information systems where either spatial interfaces or timeline-like graphics helped visualise architectural changes [8][9]. But at the end of the day, although our KR effort did integrate time and space, means to perform visual analysis privileged either time or space. In this research we present an experimental visualisation- called concentric time - designed according to four simple ideas:

- a combination of spatial features and chronology allowing comparisons enforced within the eyespan [10],

- $\quad$ an application of the context+focus principle,

- a support for uncertainty assessment (fuzziness, impreciseness, lacks, etc),

- a simple (in the sense of [11]) visualisation minimising the learning curve and the decoding effort, facilitating information discovery (including by a public of non-experts).

It is applied and evaluated on the market square in Krakow, Poland, a 200*200m urban space where 24 artefacts have been built, modified, and for most of them destroyed, over a period of 750 years. Primarily designed as a visualisation, it also acts as an interface. Section 2 details where we start from. Section 3 presents the concentric time visualisation itself. In section 4 , its evaluation is discussed. Finally, in sections 5 and 6 , we point out some of its limits, and further possible investigations.

${ }^{1}$ Classic gothic cathedrals in Bourges or Chartres were built in some decades, starting from the end of the XIIth century. The construction of the gothic cathedral in Tours started at the same period, but lasted until the XVIth century. And it is precisely that difference in time that explains some of the differences in the shapes of these edifices, i.e. differences in space.

${ }^{2}$ The information can be imprecise to the extent that although an edifice $\lambda$ did exist between time $\boldsymbol{t} \boldsymbol{1}$ and $\boldsymbol{t} \boldsymbol{2}$ in a given "area", we cannot precisely position it inside this area. However, un-localised e may have had an influence on others around. For instance, in our case study, an edifice called "Smatruz" (an open market hosting traders temporarily), localised "probably somewhere in the south west corner", was destroyed in mid XVth century. Its function being still needed, it is apparently drifted to nearby "Sukiennice", causing changes on this latter edifice, i.e. no reasoning on "Sukiennice" without representing un-localised "Smatruz". 


\section{Research background}

Combining spatial information and a chronology inside one unique representation is far from being a new problem. In a way, it is a feature of Roman "ribbon maps", where localities across the empire are connected through lines that mean travel durations, and not actual distances. Far before the "computer era", XIXth century scientists developed inventive and sometimes brilliant solutions like Minard's "figurative maps", to this day still regarded as exemplary [12]. John Snow's analysis of the 1854 London cholera epidemic, as masterly analysed by E.R Tufte [10], shows how reasoning both on time and space, in a casual manner, can been vital. Yet we today expect from graphics features that paper-based solutions cannot offer (updatability, interactive browsing, interface capabilities, etc.). Let us still remember to counterbalance the natural verbosity and jumble-hungriness of computer-based solutions with the clarity of mind of the above mentioned precursors.

In this section we briefly present some of the prominent solutions to combine space and time explicitly, at visualisation level. Our intent is not exhaustiveness - unreachable here (see for instance [4]) - but to say basically where we start from.

Originating from geosciences for the former, from engineering sciences for the latter, GIS platforms and CAD tools can allow users to display at a given position $\boldsymbol{p}$ successive versions of an object using the "layer" concept (although this concept may take a different name depending on the actual software). And indeed, the layering of time-related info may be efficient in terms of information delivery. Each version of the object can correspond to a given time slot, and so the job is done - space and time are present. Well apparently the job is done, but apparently only: when giving a closer look time does not really exist here as such (with for instance vital parameters such as durations of changes not present). Versioning offers a focus view, not a context view: what you get to see are moments in an object's evolution, hardly its whole history. A number of interesting research works introduce, at modelling level, ideas to help overcoming weaknesses inherent to the abovementioned solutions, like [13]. Nevertheless, GIS-inspired solutions have found a wide audience among archaeologists, with a recurrent trend to try and implement "4D" information systems about the history of a site [14] [15]. But GIS and CAD tools are basically concerned with distributing things in space. Consequently, $\mathbf{x}$ and $\mathbf{y}$ axes of the representation are requisitioned for spatial data - and time has to manage with what is left. Time geography may have started from the same observation - the $\mathbf{z}$ axis is here used to superimpose on a basic $\mathbf{x , y}$ cartography movements across a territory [16]. But the primary goal of time geography is to record and represent movements occurring in space over short periods of time. In our case artefacts do not "move" (or rarely...): they just change, and over long periods of time. Time geography is a promising concept - its adequacy to represent architectural changes remains questionable.

Widespread in information sciences, timelines, time bars, time charts are used to represent dates, periods, etc. A wide attention is notably put on the issue of visualising time-related phenomena in the field of information visualisation (see for instance [1], [10]). Such graphics do help reading a chronology, with events, durations, and possibly uncertainty assessments. A number of generic solutions have been developed in the past years, with some convincing results like [17]. But because time is represented 
in space - typically with a line or a spiral - spatial features tend to be scattered here and there, causing visual discomfort rather than helping to understand spatial changes. The chronographs experience [9] taught us that although a timeline-like visualisation does greatly enhance reasoning about architectural changes, it is not well suited to spatial reasoning.

With computer implementations, interactive time sliders have been successfully introduced in many research and/or communication works [8]. As mentioned before, time sliders allow users to choose time slots they are interested in, and investigate the corresponding spatial configuration. But paradoxically, time sliders are mainly efficient in helping us to understand spatial changes. In fact, they are fundamentally a mean to browse through versions of objects using time as selection criteria, and in no way a context+focus visualisation. In a recent experiment we added to this time slider mechanism a visualisation of densities of changes: this context view however only focuses on time aspects - spatial changes remaining readable only one at a time.

As alternative, visual metaphors can be proposed that link spatial changes and a chronology - T.Ohta presents some nice concepts in [19]. In previous works, we developed a 3D metaphor called Infosphere, and a 2D metaphor called "ladybug race" that were equally "enjoyable". But neither the former (no durations, 3D navigation disconcerting for some users) nor the latter (no neighbourhood relations) did solve the problem. And so at the end of the day we are left with a number of solutions that correspond only in part to what we expect from a visualisation of dynamics of change.

The concentric time visualisation can be seen as coming to an arrangement between some of these solutions. It capitalizes on basic, well-known, well understood representations (cartography, timelines). It differs from them by introducing a specific, context+focus, graphic combination of spatial features and chronology. It is designed to help researchers reconsider their knowledge and the doubts that travel with it, and puts space and time on equal terms.

\section{The concentric time visualisation: principles, implementation}

To start with, let us introduce some of the terms that we will use in this section:

- the evolution of an artefact is the time span separating its creation from its extinction (i.e. full physical removal, including of sub-structures, [9]);

- morphological changes imply transformations of the artefact's "shape",

- recurrent changes may concern upkeep, ownership, function, etc.,

- contours are simplified representations of an artefact's plan,

- uncertainty may concern the dating of events, the very existence of events, or the physical reality of an artefact. In all cases it is a consequence of the evidence we base on. It is represented in this experiment by a simplified lexical scale (known, uncertain, hypothetical).

Figures below present the visualisation's construction as a series of steps introducing spatial or temporal information - these steps should not be interpreted as "successive actions inside the construction procedure". The left part of figures illustrates the idea, the right part what it looks like when applied to our real case (for 
enhanced readability of the paper output, original contrast and line thicknesses of the computer output are accentuated). draw
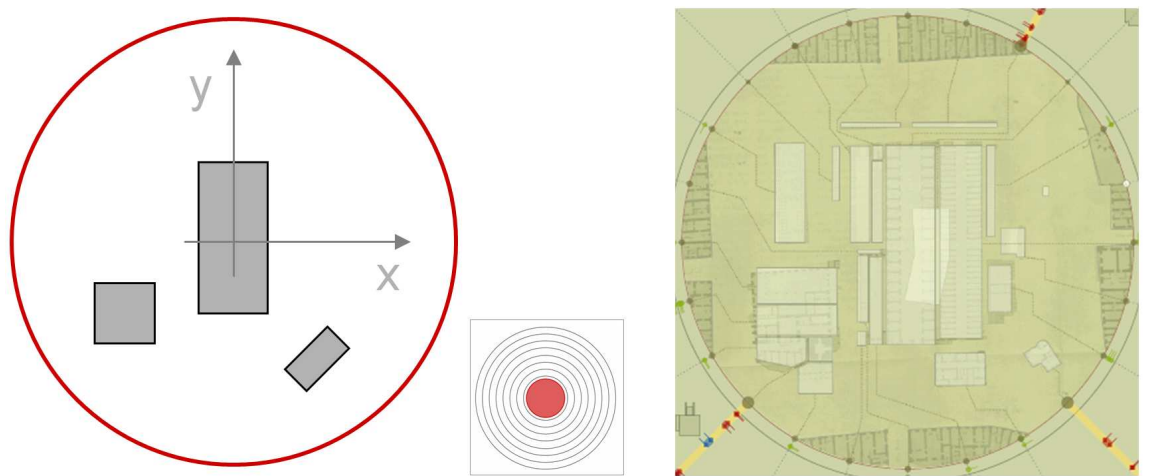

Fig. 1. A 2D map is displayed inside a red circle, showing simplified outlines of the artefacts under scrutiny. These contours are there to get an idea of where the artefacts were located, but they are not exact contours ${ }^{3}$. With no reasonable justification, an old map acts as a background.

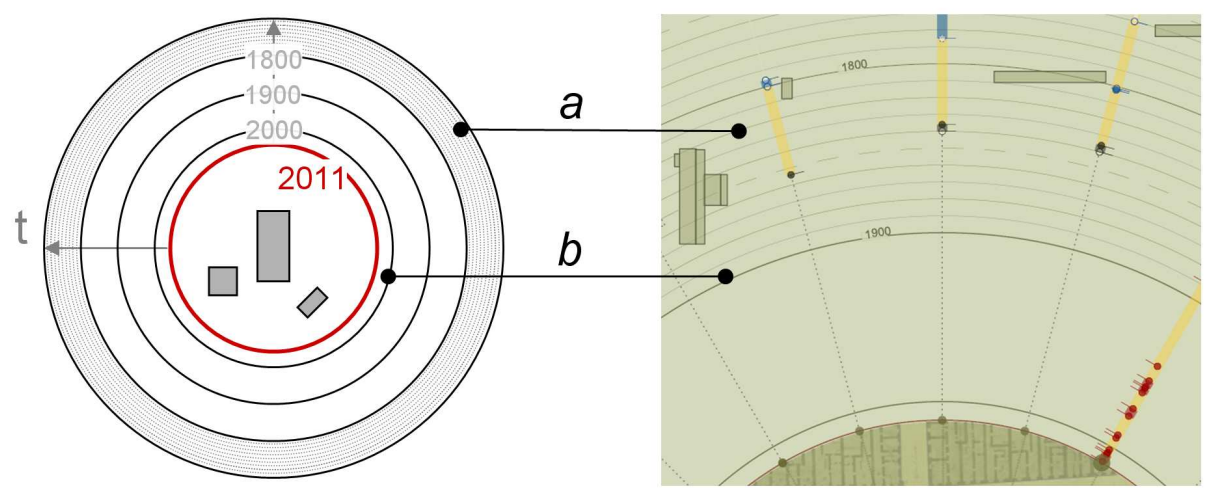

Fig. 2. Starting from the red circle (that stands for the current year - 2011), concentric circles represent a move towards the past. A circle is drawn for each decade (a) or century $(\boldsymbol{b})$, except during the XXth century (this for readability purposes, considering very few changes occur then in our case study). The farer a circle is from the center, the older it is.

${ }^{3}$ There are several good reasons to this choice:

- A number of artefacts were built over another, older one - in other words at the same x,y position. Drawing contours one over the other would result in a good degree of visual pollution. Accordingly contours are represented in such a way as to minimise overlapping.

-If we were to represent a given version of an artefact on loading of the system, we would have to privilege one version of an artefact over another - and there is no reason to do so. Furthermore, if we were to privilege version 1 for artefact $\lambda$ and version 3 for artefact $\delta$, we could end up with showing side by side edifices that never were there at the same moment. So that means we should rather select a given moment in history - well in that case we would just not see the whole set of contours since they never were all present at the same time.

In short, showing approximate contours enhances readability, and avoids spatial or temporal inconsistency. 


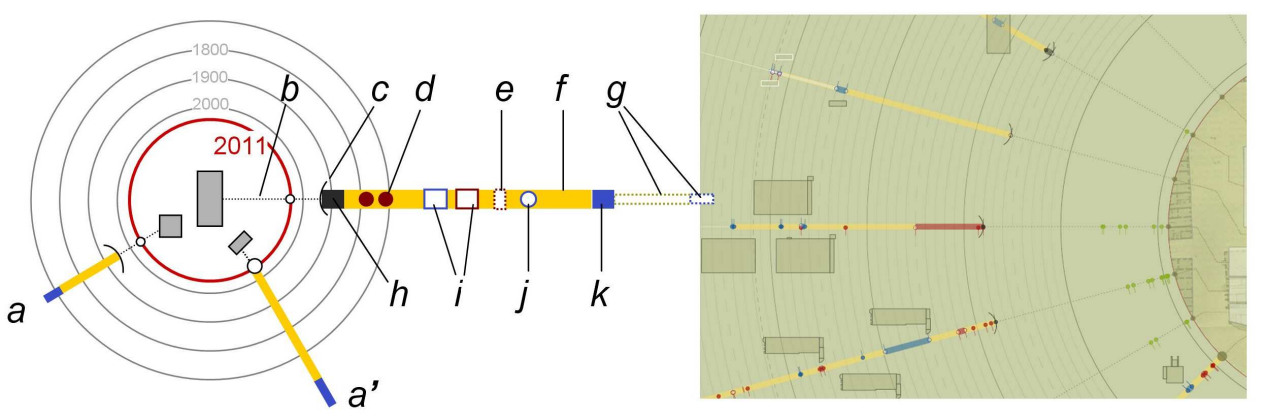

Fig. 3. Artefacts are connected to radial timelines $\left(\boldsymbol{a}, \boldsymbol{a}^{\prime}\right)$ that distributes events in time and recount changes through two graphic variables:

- Four colours : yellow (periods of stability, no known change, $\boldsymbol{f}$ ), blue (morphological change, including construction, $\boldsymbol{k}$ ), red (recurrent change, $\boldsymbol{d}$ ), grey (destruction, $\boldsymbol{c}$, meaning here that no visible trace is left above the ground).

- Shapes: differentiate long-lasting events (duration > year, represented by rectangles $-\boldsymbol{I}, \boldsymbol{e}, \boldsymbol{k}$ ) from punctual events (duration < a year, represented by a circle $-\boldsymbol{d}, \boldsymbol{j}$ ). When the dating of a period is uncertain, coloured rectangles as well as circles are filled in white, and only outlined in colour $(\boldsymbol{i}, \boldsymbol{j})$. When the dating of a period is hypothetical, the outlined is dashed $(\mathbf{e}, \mathbf{g})$. When the very existence of an artefact is uncertain, the size of coloured rectangles is modified (halfwidth, $\boldsymbol{g}$ ).

Visible on the right figure, an arc marks an artefact's destruction (c). It is withdrawn from the representation when zooming in, in order to lower the overall graphic weight. Timelines for artefacts that remain up to now are continued up to the central circle $\left(\boldsymbol{a}^{\prime}\right)$. Capitalizing on the graphics' radial structure, dotted grey lines connect the timeline to a position on the central circle; white lines extend short timelines backwards in time $(\boldsymbol{b})$.
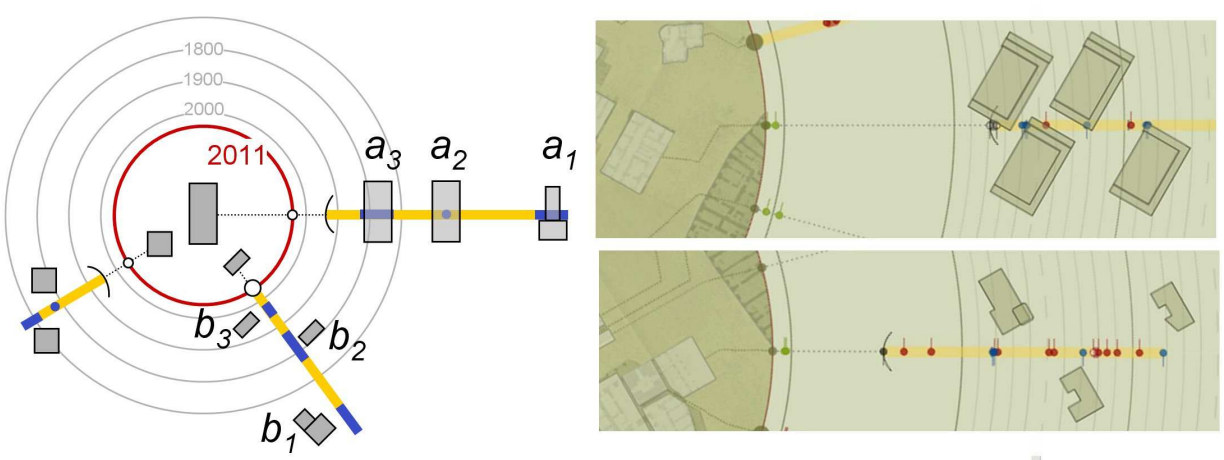

Fig. 4. All along the timeline, a new contour is drawn for each "blue" (morphological) transformation. The contour is drawn either on the timeline or above/below the timeline (a point we re-discuss in the implementation section).

Depending on what really happened, the actual geometry of the new contour may be left unchanged from version to version ( $\boldsymbol{a}$ - adding a new storey for instance does not change the artefact's contour). However, the visualisation acts also an interface, each contour is connected to queries that are specific to it - allowing users to retrieve info (basic data, 2D/3D content, bibliography, etc.) about what really happened (this point is re-discussed in the perspective section). The timelines radiate around the central circle, but the contour's orientation to the north is maintained whatever angle the timeline is drawn at. 

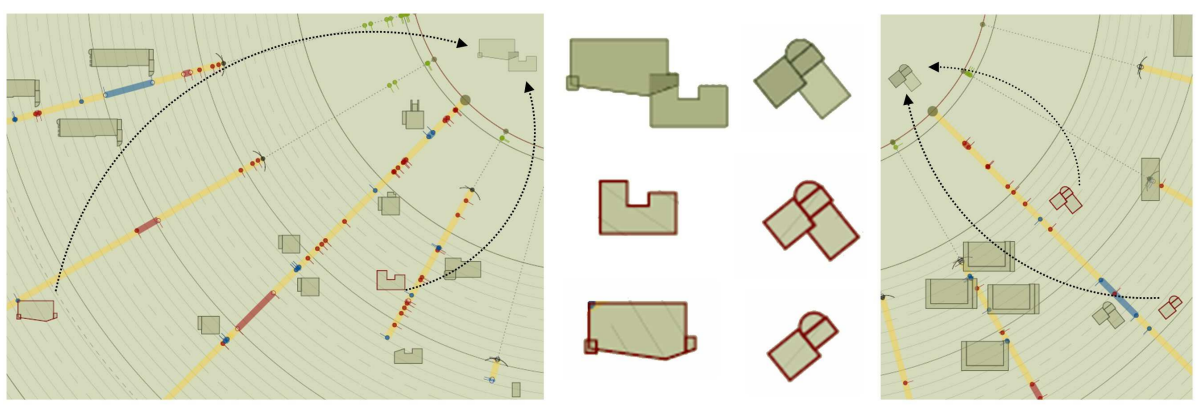

Fig. 5. Finally, in order to allow basic spatial analysis, each of the contours can be projected inside the central circle on user selection. Because contours are drawn with a level of transparency, this mechanism enables user-monitored comparisons of contours at two levels:

- Left, Neighbourhood analysis - in order to uncover the likelihood of event propagation - fires for instance, a plague at those periods.

- Right, Transformation analysis - shows the density of use of the ground over time, or helps spotting the period of emergence of a given component, and check on neighbouring artefacts whether "there is room for the added component" at that moment in history.

When contours are projected, the central circle's content is simplified (background image is hidden as well as simplified contours) so as to avoid visual overload.

The concentric time visualisation has been applied to the evolution of Krakow's Main Square (24 artefacts, 79 "contours", 391 "events" over 750 years). Although we already knew quite well this site, the visualisation did help us spot some interesting patterns - like an unexpected pattern of uncertainty on XIXth century destructions in the north-west corner. The following figures illustrate on two examples how the visualization may support reasoning tasks.
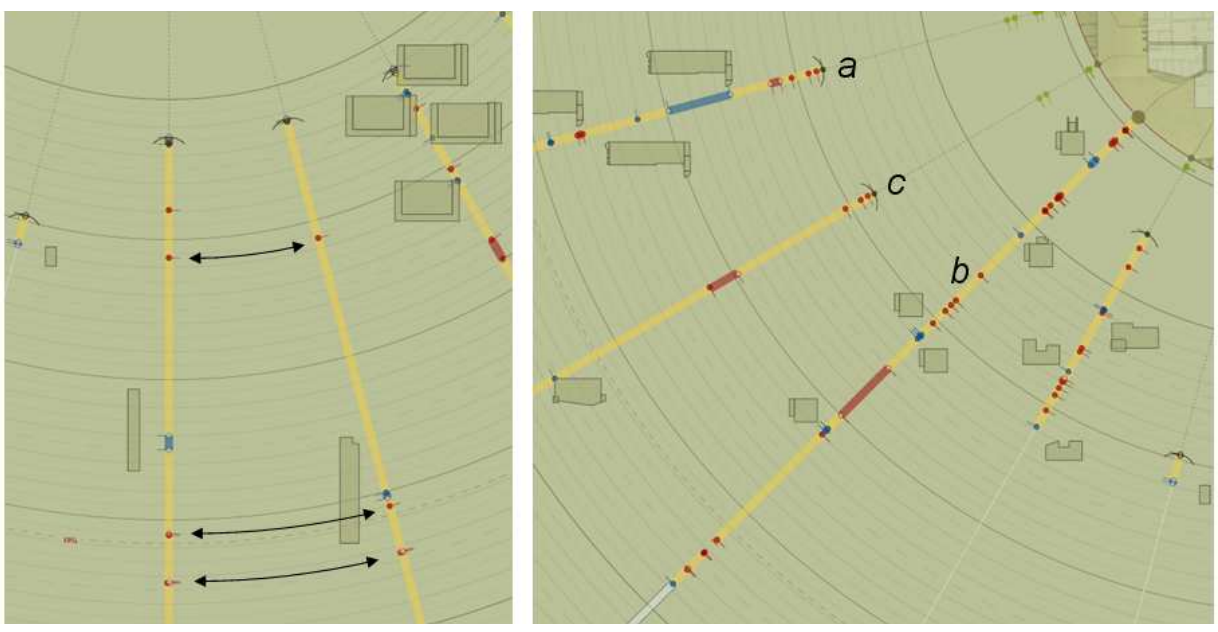

Fig. 6. Left, a focus view helps analysing causal relations between events and changes on neighbouring objects note parallel recurrent changes in both artefacts (red dots) independently of morphological changes. Right, a focus view allows comparisons of patterns between objects: compare densities of changes for $(\boldsymbol{a}),(\boldsymbol{b})$ to this of $(\boldsymbol{c})$ (a prison). 


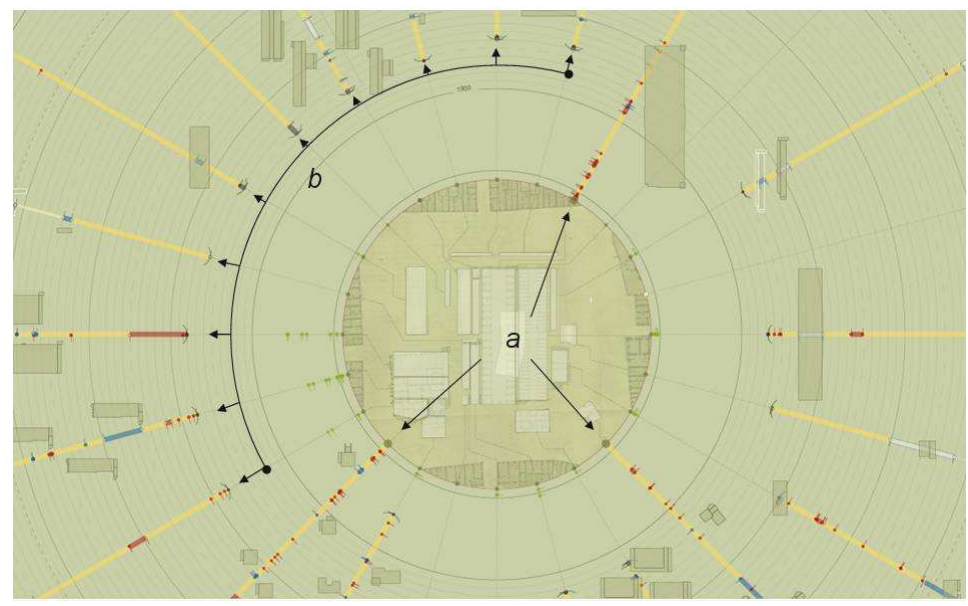

Fig. 7. Left, context view underlines features of the collection: for instance the proportion of remaining artefacts (a), 3 out of 24 , and the impact of mid XIXth century destructions (b).

The implementation is a fairly primitive one, combining two main levels:

- Reusable components, composed of an RDBMS where information for each artefact are stored (dating, sources, description of each of the artefact's change) and of XML files (geometrical information for each version of an artefact. These components, developed in previous experiments [8][9], were here only updated with some recent archaeological evidence.

- The visualisation itself - an SVG file [18] produced on the fly for standard web browsers (Perl classes - architectural ontology - and modules developed in the abovementioned experiments), with user interactions monitored inside Javascript modules (zoom/Pan, show/hide, contour projection, queries, etc.).

Finally, the visualisation also acts as an interface: specific menus are available either over contours or over events that allow the querying of various data sets (RDBMS, $\mathrm{XML}$ files, as well as static 3D models corresponding to each contour).

\section{Evaluation, limitations, perspectives}

The evaluation was carried out with a group of eleven students in mechanical engineering (no background in architecture or historic architecture, no knowledge about the case study). Willingly, the session lasted less than 30 minutes, including the time needed for us to present the visualisation's objective and graphic codes. The visualisation was projected on a white screen; with zoom levels fitted to the various questions.

\footnotetext{
${ }^{4}$ A puzzling problem was how to position contours along the timeline (across, above, below? - see Fig4.). No systematic solution appeared as satisfactory. For each contour we specify manually, inside a javascript "configuration" file, an x,y move from its "natural" position (which is the center of a blue rectangle / circle). The visualisation is dynamically written with "natural" positions, and an onload jscript event moves the contours according to the configuration file. Contours can also be freely re-positioned by users (mouse dragging).
} 
Testers were asked 14 questions distributed into 5 groups (space reading, chronology, uncertainty, comparing/analysing individuals, correlating space and time).

In the first three groups of questions we tested the visualisation's readability: testers were asked to decode the visualisation and to write down "how long does transformation $\mathbf{t}$ lasts", "which in this group of artefacts does not fit", etc. Facing questions of the two last groups, testers had to do some reasoning with questions like "identify pattern $\boldsymbol{p}$ ", "can modification on artefact $\lambda$ be related to events in the neighbourhood", etc.

Results show an error rate of 3\%, mostly on readability questions. With such a low error rate, our questionnaire may be considered as not demanding enough. But answers were easy to give provided the visualisation was easily and unambiguously understood: and this is precisely what we were eager to verify. The evaluation does indicate the visualisation itself is almost self-evident, and moreover that some domainspecific notions (levels of credibility or uncertainties in particular) were correctly grabbed by full beginners in the field. We make no other claim about the evaluation. What has to be said is that the visualisation is a result of the interpretation of more than 400 bibliographic sources. Accordingly an evaluation that would allow comparing "reasoning from sources" and "reasoning with the visualisation" is far from being easy to build. But it is clear that we do not consider this evaluation as a definitive one. A number of issues remain open, for which future works will be needed:

- Case-study bias. The spatial layout of artefacts considered is our case study is well suited to the visualisation (artefacts kept close to one another for instance). A more complex spatial configuration, with more artefacts, with artefacts scattered in a wider area, or with more complex overlapping, may result in less convincing visual results. Tests on other case studies should be considered.

- Some additional features would definitely improve the visualisation's usability: independent zooming on spatial/temporal data, multi-resolution and partial zooming on timelines for short events, alternative levels of details for contours, etc. Our objective was not to come out with a ready-to-use system, but to test a concept: it is possible that this concept may be better implemented with a different technical set.

- In this experiment, only contours of artefacts are offered for users to carry out spatial analysis. Spatial analysis should be broadened to other 2D/3D characteristics.

- What the visualisation really does is nothing more than placing time and space side by side, and having them interact. Whether a deeper integration would bring better results remains to be established.

\section{Conclusion}

We introduce a context+focus visualisation called concentric time aimed at summarising the journey through time of groups of artefacts. The visualisation can be used for research purposes, but was evaluated with as ulterior motive testing its usability for a wider public, with possible museology applications. It was designed in order to combine inside a unique information space, spatial features and chronology, with as constraints uncertainty assessment, interface capabilities, and simplicity. The concentric time visualisation has been applied and evaluated on a case study, thanks to which 
we checked its support in carrying out reasoning tasks about architectural changes. Its limitations are numerous: what were investigated here really are the possible benefits of the concept- no claim is made on a generic, ready to use, system. On the other hand, the concept appears as fairly generic: its usability beyond clarifying architectural changes could be investigated (for instance in geoinformation). Providing models and visual tools to handle dynamics of change remains today a hot research topic: we view our contribution as demonstrating that besides facing sometimes complex knowledge modelling challenges, researchers in the field also face the challenging complexity of simple visual thinking.

\section{References}

1. Spence, R.: Information vizualisation. Addison Wesley ACM Press, Essex (2001).

2. Bertin, J.: Sémiologie graphique, EHESS, Paris (1998).

3. Card, S.K., Mackinlay, J.D, Schneiderman, B.: Information vizualisation: Using vision to think. Morgan-Kaufmann, San Francisco (1999).

4. Sanders, L (Ed) :Models in Spatial Analysis London; Newport Beach, CA : ISTE (2007).

5. Dürstener, J.C.: Visualising Time. <http://www.infovis.net/ > (accessed feb2011) (2006).

6. Jensen, M.: Semantic Timeline Tools for History and Criticism, In Digital Humanities (June 2006) 97-100.

7. Kienreich, W.: Information and Knowledge Visualisation: an oblique view, MiaJournal, Vol. 0, (2006) 7-16.

8. Blaise, J.Y, Dudek, I.: From artefact representation to information visualisation: genesis of informative modelling. Lecture Notes in Computer Science, Vol. 3638. Springer-Verlag, Berlin Heidelberg New York (2005) 230-236.

9. Blaise, J.Y, Dudek, I.: Profiling artefact changes: a methodological proposal for the classification and visualisation of architectural transformations, Digital Heritage, Archeolingua, Budapest (2008) 349-356.

10. Tufte, E.R.: Visual explanations. Graphic Press, Cheshire (1997).

11. Maeda, J.: No simplicity without complexity, In G.Schuller, Designing universal knowledge, Lars Muller Publisher (2008) 138-143.

12. Friendly, M.: Re-visions of Minard, Statistical computing and graphics newsletter, Vol 11, $\mathrm{n}^{\circ} 1(1999)$

13. Rodier, X., Saligny L.: Social features, Spatial features, Time features : An urban archaeological data model, In Posluschny A., Lambers K., Hertog I. (eds.), Proc. CAA, ( 2007).

14. Johnson, I.: Mapping the fourth dimension: the TimeMap project. In Dingwall, L. et al. Archaeology in the Age of the Internet. British Archaeological Rep. Int. Series 750 (1999).

15. Van Ruymbeke M., et al, : Development and use of a 4D GIS to support the conservation of the Calakmul site. Proc. 1st International EARSeL Workshop, Lasapona, R Masini, N (Eds) Aracne (2008) 333 - 338.

16. Yu, H., Shaw S-L.: Revisiting Hägerstrand's Time-Geographic Framework for Individual Activities in the Age of Instant Access. Proc. Research Symposium Salt Lake City (2005) 103-118.

17. Sabol, V., Scharl, A.: Visualizing Temporal-Semantic Relations in Dynamic Information Landscapes. In AGILE 2008 Int. Conf. on Geographic Information Science (2008).

18. Rathert, N.A., 2005. Knowledge visualisation using dynamic SVG Charts, In Geroimenko, V., Chen C (Eds).: Visualizing information using SVG and X3D, Springer (2005) 245-254.

19. T.Ohta.: Diagram design course, In Informational Diagram Collection, Pie Books Tokyo (2009) 213-222. 